\title{
ORDER CONVERGENCE OF ORDER BOUNDED SEQUENCES IN RIESZ SPACES
}

\author{
BORIS LAVRIČ
}

\begin{abstract}
We consider sequences $\left(x_{n}\right)_{n=1}^{\infty}$ in a Dedekind $\sigma$-complete Riesz space, satisfying a recursive relation

$$
x_{n+p} \geq \sum_{j=1}^{p} \alpha_{n j} x_{n+p-j} \quad \text { for } n=1,2, \cdots
$$

where $p$ is a given natural number and $\alpha_{n j}$ are nonnegative real numbers satisfying $\sum_{j=1}^{p} \alpha_{n j}=$ 1. We obtain a sufficient condition on coefficients $\alpha_{n}$; for which order boundedness of 8 uch, a sequence $\left(x_{n}\right)_{n=1}^{\infty}$ implies its order convergence. In a particular case when $\alpha_{n j}=\alpha_{j}$ for all $n$ and $j$, it is shown that every order bounded sequence satisfying the above recursive relation order converges if and only if natural numbers $j \leq p$ for which $\alpha_{j}>0$, are relative prime.
\end{abstract}

\section{Introduction}

Throughout this paper we denote by $N$ the set of natural numbers and by $\mathbb{R}$ the set of real numbers. For notation, terminology and basic results on Riesz spaces (vector lattices) we refer the reader to [1] and [2]. We recall here some definitions. A sequence $\left(x_{n}\right)_{n=1}^{\infty}$ in a Riesz space $L$ is said to be increasing (respectively decreasing) if $x_{n} \leq x_{n+1}$ (respectively $x_{n+1} \leq x_{n}$ ) for all $n \in \mathrm{N}$; it is order bounded if there exists an element $y \in L$ such that $\left|x_{n}\right| \leq y$ for all $n \in \mathrm{N}$, and order convergent if there exists an element $x \in L$ such that $\left|x_{n}-x\right| \leq v_{n}$ for some decreasing sequence $\left(v_{n}\right)_{n=1}^{\infty}$ satisfying $\inf _{n \in N} V_{n}=0$. A Riesz space $L$ is said to be Dedekind $\sigma$-complete if for every increasing order bounded sequence $\left(x_{n}\right)_{n=1}^{\infty}$ in $L$ there exists $\sup _{n \in \mathbb{N}} x_{n}$. It is easy to see that a Riesz space $L$ is Dedekind $\sigma$-complete if and only if every order bounded increasing sequence in $L$ order converges.

In this note we study order convergence of order bounded sequences $\left(x_{n}\right)_{n=1}^{\infty}$ satisfying a recursive relation

$$
x_{n+p} \geq \sum_{j=1}^{p} \alpha_{n j} x_{n+p-j} \quad \text { for } n=1,2 \ldots
$$

Received November 231996.

1991 Mathematics Subject Classification. 06F20,40A05.

Key words and phrases. Riese space, order convergence, sequence. 
where $p \in N, 0 \leq \alpha_{n j} \in \mathbb{R}$, and $\sum_{j=1}^{p} \alpha_{n j}=1$. We obtain a sufficient condition on coefficients $\alpha_{n j}$ for which order boundedness of such a sequence $\left(x_{n}\right)_{n=1}^{\infty}$ in a Dedekind $\sigma$-complete Riesz space implies its order convergence. In a particular case when $\alpha_{n j}=\alpha_{j}$ for all $n$ and $j$, it is shown that every order bounded sequence satisfying the above recursive relation order converges if and only if natural numbers $j \leq p$ for which $\alpha_{j}>0$, are relative prime.

Results. In order to shorten the formulation of our main result let us introduce the following set of functions. If $p \in \mathbb{N}$ and $\alpha_{n j} \in \mathbb{R}$, where $n=1,2 \ldots$ and $1 \leq j \leq p$, then for every triple $(\beta, m, n) \in \mathbb{R} \times \mathrm{N} \times \mathrm{N}$ denote by $S_{\beta}(m, n)$ the set of all functions $f:\{0,1, \cdots, m\} \rightarrow N$ such that $f(0)=n$ and

$$
1 \leq f(k+1)-f(k) \leq p, \quad \alpha_{f(k+1), f(k+1)-f(k)} \geq \beta
$$

for all nonnegative integers $k<m$.

Theorem. Let $L$ be a Dedekind $\sigma$-complete Riesz space, and $\left(x_{n}\right)_{n=1}^{\infty}$ and order bounded sequence of its elements such that

$$
x_{n+p} \geq \sum_{j=1}^{p} \alpha_{n j} x_{n+p-j} \quad \text { for all } n \in \mathbb{N},
$$

where $p$ is a given natural number and $\alpha_{n j}$ are nonnegative real numbers satisfying $\sum_{j=1}^{p} \alpha_{n j}=1$ for all $n \in \mathrm{N}$. If there exist a real number $\beta>0$ and natural numbers $M, N$ such that for every $n \geq N$ the set

$$
R_{\beta}(M, n)=\left\{f(k): f \in S_{\beta}(m, n), 0 \leq k \leq m \leq M\right\}
$$

contains $p$ consecutive natural numbers, then the sequence $\left(x_{n}\right)_{n=1}^{\infty}$ is order convergent.

Proof. Suppose that the numbers $\alpha_{n j}$ and the sequence $\left(x_{n}\right)_{n=1}^{\infty}$ satisfy all conditions of the theorem. Since $\left(x_{n}\right)_{n=1}^{\infty}$ is order bounded, the Dedekind $\sigma$-completeness of $L$ implies that there exist elements

$$
x=\sup _{n \in N} \inf _{j \geq n} x_{j}, \quad y=\inf _{n \in N} \sup _{j \geq n} x_{j} .
$$

Obviously $x \leq y$, and we have to show that $x=y[2]$. Suppose by contradiction that $x<y$ and assume without loss of gencrality that $x=0$ (replacing $x_{n}$ by $x_{n}-x$, if necessary). Let $\epsilon>0$ and observe that there exists $m \in \mathrm{N}$ such that

$$
u=\left(\inf _{j \geq m} x_{j}+c y\right)^{+}>0 .
$$

If there exists $k \in \mathbb{N}$ such that $x_{j} \wedge y \leq-u$ for all $j \geq k$, then $\sup _{j \geq k} x_{j} \wedge y \leq y-u$ which contradicts the definition of $y$. Hence, there exists a natural $n \geq \max \{m, \bar{N}\}$ such that

$$
v=\left(x_{n+p} \wedge y+u-y\right)^{+}>0 \text {. }
$$


If $P$ is the projection onto the band of $L$ generated by $v$, then $P\left(x_{n+p} \wedge y+u-y\right)=v$. Using also the inequality $u \leq \epsilon y$, it can be seen easily that

$$
P x_{n+p} \geq v+(1-\epsilon) P y .
$$

If $i \geq m$, then $\left(x_{i}+\epsilon y\right)^{+} \geq u$ and therefore $\left(x_{i}+\epsilon y\right)^{-} \wedge u=0$. Since $0<v \leq u$, this implies $\left(x_{i}+\epsilon y\right)^{-} \wedge v=0$. It follows that $P\left(x_{i}+\epsilon y\right)^{-}=0$ and consequently $P\left(x_{i}+\epsilon y\right) \geq P u \geq v$, hence

$$
P x_{i} \geq v-\epsilon P y \quad \text { for all } i \geq m
$$

We claim that

$$
P x_{f(k)+p} \geq v+\left(\beta^{k}-\epsilon\right) P y
$$

for all $f \in S_{\beta}(m, n)$ and $k \in\{0, \cdots, m-1\}$. Indeed, it follows from (2) that (4) holds for $k=0$. We proceed the proof by induction on $k$. Assume that (4) holds for some nonnegative $k<m-1$. Using (1) and (3) we get

$$
\begin{aligned}
P x_{f(k+1)+p} & \geq \sum_{j=1}^{p} \alpha_{f(k+1), j} P x_{f(k+1)+p-j} \\
. & \geq \alpha_{f(k+1), f(k+1)-f(k)} P x_{f(k)+p}+\left(1-\alpha_{f(k+1), f(k+1)-f(k)}\right)(v-\epsilon P y) \\
& \geq v+\left(\alpha_{f(k+1), f(k+1)-f(k)} \beta^{k}-\epsilon\right) P y \geq v+\left(\beta^{k+1}-\epsilon\right) P y
\end{aligned}
$$

and the induction step is complete.

By assumption there exist $p$ consecutive numbers

$$
f_{j}\left(k_{j}\right)=l+j, \quad j=1, \cdots, p,
$$

where $f_{j} \in S_{\beta}\left(m_{j}, n\right)$ and $0 \leq k_{j} \leq m_{j} \leq M$ for $j=1, \cdots, p$. Note that $0<\beta \leq 1$, and take $\epsilon=\beta^{M}$. The inequality (4) implies that

$$
P x_{l+j+p} \geq v+\left(\beta^{k_{j}}-\epsilon\right) P y \geq v
$$

holds for $j=1, \cdots, p$. Using (1) we get easily $P x_{i} \geq v$ for all $i>l+p$. It follows that $\sup _{n \in \mathbb{N}} \inf _{j \geq n} P x_{j} \geq v$, which contradicts the equality $\sup _{n \in \mathbb{N}} \inf _{j \geq n} x_{j}=x=0$.

The above theorem can be modified a little if a sequence $\left(x_{n}\right)_{n=1}^{\infty}$ satisfies

$$
x_{n+p}=\sum_{j=1}^{p} \alpha_{n j} x_{n+p-j} \quad \text { for all } n \in \mathbb{N} .
$$

Namely, in this case $\inf _{1 \leq j \leq p} x_{j} \leq x_{n} \leq \sup _{1 \leq j \leq p} x_{j}$ holds for all $n \in \mathbb{N}$, hence the requirement of order boundedness is superfluous in the theorem.

The next result gives a sufficient condition for coeficients $\alpha_{n j}$ to satisfy the requirement of the theorem. 
Proposition. If there exists a subset $J \subseteq\{1, \cdots, p\}$ which consists of $k$ relatively prime numbers $j_{1}, \cdots, j_{k}$ and satisfies

$$
\beta=\inf \left\{\alpha_{n j}: n \in N, j \in J\right\}>0,
$$

then there exists $M \in N$ such that for every $n \in N$ the set $R_{\beta}(M, n)$ contains $p+1$ consecutive numbers.

Proof. Let $n \in N$. If $f:\{0,1, \cdots, m\} \rightarrow N$ satisfies $f(0)=n$ and $f(k+1)-f(k) \in J$ for all nonnegative integers $k<m$, then by $(5)$ we have $f \in S_{\beta}(m, n)$. It follows that the set $\left\{f(k): f \in S_{\beta}(m, n), 0 \leq k \leq m\right\}$ consists of all numbers of the form

$$
n+\sum_{i=1}^{k} m_{i} j_{i}, \quad 0 \leq m_{i} \in \mathrm{Z}, \quad \sum_{i=1}^{k} m_{i}=m .
$$
Since $j_{1}, \cdots, j_{k}$ are relatively prime, there exists integers $l_{1}, \cdots, l_{k}$ such that $\sum_{i=1}^{k} l_{i} j_{i}=$
1. Put

$$
l=\max _{1 \leq i \leq k}\left|l_{i}\right|, \quad M=2 k p l
$$

and note that for each nonnegative integer $q \leq p$ we have

$$
m_{i}(q)=q l_{i}+p l \geq 0, \quad \sum_{i=1}^{k} m_{i}(q) \leq M .
$$

It follows that for each $n \in N$ the set $R_{\mathcal{B}}(M, \eta v)$ contains numbers

$$
n+q+p l \sum_{i=1}^{k} j_{i}=n+\sum_{j=1}^{k} m_{i}(q) j_{i}
$$

where $q=0,1, \cdots, p$.

The proof of the above proposition can be shortened if we use the fact that the additive subsemigroup $S(J)$ of $N$ generated by a subset $J=\left\{j_{1}, \cdots, j_{k}\right\}$ with relativcly prime numbers $j_{1}, \cdots, j_{k}$, has finite complement in $N$. Indeed, since $S(J)$ consists of all sums of the form $\sum_{i=1}^{k} m_{i} j_{i}, 0 \leq m_{i} \in \mathbb{Z}$, there exists $M \in N$ such that for every $n \in N$ the set $R_{\beta}(M, n)$ has finite complement in $N$.

In a special case of the above theorem and proposition we get the following result.

Corollary. Let $L$ be a nontrivial Dedekind $\sigma$-complete Riesz space, and $\left(x_{n}\right)_{n=1}^{\infty} a$ sequence of its elements such that

$$
x_{n+p} \geq \sum_{j=1}^{p} \alpha_{j} x_{n+p-j} \quad \text { for all } n \in N,
$$

where the nonnegative real numbers $\alpha_{j}$ satisfy $\sum_{j=1}^{p} \alpha_{j}=1$. Then the following state-
ments are equivalent. 
(i) The natural numbers $j \leq p$ satisfying $\alpha_{j}>0$ are relatively prime.

(ii) The sequence $\left(x_{n}\right)_{n=1}^{\infty}$ is order convergent if and only if it is order bounded.

Proof. Take $\alpha_{n j}=\alpha_{j}$ for each $n \in N$ and $j \in\{1, \cdots, p\}$, and put

$$
J=\left\{j \in N: 1 \leq j \leq p, \alpha_{j}>0\right\}
$$

If (i) holds, then $J$ satisfies the condition of the proposition, hence (ii) follows from the theorem.

If (i) does not hold, then the greatest common divisor $m$ of the numbers from $J$ is greater then 1 . For a nonzero $a \in L$, the sequence $\left(x_{n}\right)_{n=1}^{\infty}$ defined by

$$
x_{n}= \begin{cases}a & \text { if } m \mid n \\ 0 & \text { otherwise }\end{cases}
$$

satisfies (6). Since it is order bounded but not order convergent, (ii) does not hold.

An inspection of the proof shows that if the sequence $\left(x_{n}\right)_{n=1}^{\infty}$ of the above corollary satisfies

$$
x_{n+p}=\sum_{j=1}^{p} \alpha_{j} x_{n+p-j} \quad \text { for all } n \in \mathbb{N},
$$

then it is order convergent if and only if the numbers of $J$ are relatively prime.

The condition that $L$ is Dedekind $\sigma$-complete cannot be dropped in the above theorem and corollary. Moreover, if $L$ is not Dedekind $\sigma$-complete, there exists an order bounded increasing sequence in $L$ which is not order convergent but obviously satisfies (1) and (6), hence the variant of the theorem and its corollary fails for such a space $L$.

\section{References}

[1] W. A. J. Luxemburg and A. C. Zaanen, Riesz Spaces I, North-Holland, Amsterdam, 1973.

[2] B. Z. Vulikh, Introduction to the Theory of Partially Ordered Spaces, Walters-Noordhoff, Groningen, 1967.

Faculty of Mathematics and Physics, University of Ljubljana, Jadranska 19,1000 Ljubljana, Slovenia. 